\title{
The Use of Human Amniotic Membrane for Cartilage Repair: A Sheep Study
}

\author{
Samuel K. Tabet, David M. Conner, Davis A. Guebert \\ New Mexico Orthopaedic Associates, Albuquerque, NM, USA \\ Email: TabetSK@nmortho.net
}

Received 25 August 2015; accepted 5 October 2015; published 8 October 2015

Copyright (C) 2015 by authors and Scientific Research Publishing Inc.

This work is licensed under the Creative Commons Attribution International License (CC BY). http://creativecommons.org/licenses/by/4.0/

(c) (i) Open Access

\section{Abstract}

Studies show that human amniotic cells' pluripotentency can be influenced to produce chondrocytes and osteocytes through adding demineralized bone (DMB). Objective: This study evaluates the human amniotic membrane (HAM) mixed with DMB to fill defects in sheep models. We hypothesized this membrane would fill these defects with hyaline-like cartilage with chondrocytes populating the matrix. Design: Six adult sheep were used in this study. One hindquarter knee of each sheep was utilized to make two cartilage defects: one on the femoral condyle and one in the trochlear grove. Three control sheep had unfilled defects. Three sheep received HAM/DMB from a placenta to fill the defects. The membrane was folded so the cellular layer faced the defect and the joint while demineralized bone was placed between the layers. The membranes were fixed to the femur and to the trochlear grove. At six months, the sheep were sacrificed for evaluation. Results: Of the controls, defects did not fill with hyaline or fibrocartilage. In HAM/DMB sheep, $50 \%$ of the defects retained the membrane, consistent with other animal models. Membrane defects were examined histologically by a validated scoring system. A strong correlation of little statistical difference between the test and the normal cartilages was observed. The defects that retained membranes had evidence of diffuse chondrocyte-like cell proliferation of stromal matrix similar to hyaline cartilage. Conclusions: HAM/DMB is a potential source of pluripotent cells that can influence chondrogenesis in defects in sheep models. The implications for application in a human model are promising and warrant further study.

\section{Keywords}

Stem Cell, Chondrocytes, Amniotic Membrane, Pluripotent, Demineralized Bone

\section{Introduction}

The repair of cartilage defects in humans can be a difficult endeavor, and multiples options exist for the surgeon 
to approach this topic. The surgeon may choose to influence the defect with microfracture or abrasion techniques to stimulate a fibrocartilage patch in which to fill the defect. Alternatively, there are also options available that allow for the filling of the defect with hyaline-like cartilage with chondrocytes in the stromal matrix of variable sources, both of autograft and allograft origin [1]. The goal with all of these procedures is to replace cartilage defects with hyaline-like cartilage or cartilage-like cells that are as close to normal hyaline cartilage as possible. It has been posited that the use of human amniotic membrane may be an ideal choice for cartilage restoration and repair as it is readily available, easy to place in a defect, and induces minimal morbidity to the patient [1].

Multiple studies exist expounding on the potential uses of human amniotic cells in various platforms for tissue repair [1]-[4]. It has been shown that amniotic cells are pluripotent in nature and can be influenced to produce various cell lines including chondrocytes [1] [5]. Furthermore, it has been shown that demineralized bone can influence pluripotent cells to produce chondrocyte and osteoblast type cells [6]. The potential for this knowledge to be used in cartilage defect repair has not been explored to the knowledge of the authors up to this point.

This study evaluates the use of human amniotic membrane (HAM) paired with demineralized bone (DMB) to fill cartilage defects in a sheep model. It was hypothesized that this HAM/DMB membrane would be able to fill defects with cell proliferation of stromal matrix similar to hyaline cartilage.

\section{Methods}

The basic design of this study was to test the efficacy of HAM/DMB membranes on two types of articular surfaces, trochlear and femoral, using six adult sheep (less than three years old) to be evaluated grossly and histologically at the end of six months. In accordance to the local Ethical Committee, all of the sheep were evaluated by a licensed veterinarian and determined to be healthy, without any limb deformity prior to this study. A licensed veterinarian anesthetized each sheep before one hindquarter knee of each was sterilized and surgically exposed. Two cartilage defects were created using curettes: one on the weight-bearing surface of the femoral condyle and one in the trochlear grove. The defects did not penetrate the subchondral bone. Three sheep were used as control sheep and the defects were left unfilled giving a total of six control defects. Three sheep were chosen to receive HAM/DMB, giving a total of six experimental grafts.

The membrane was procured from a placenta and cut to fit the defect [7]. The portion of the placenta utilized was from the amniotic membrane, which was the outer layer of the placenta without the chorion layer. This layer has been shown to have pluripotent cells [8] [9]. Care was taken to place the epithelial layer away from the bone defect, as this layer did not have pluripotent cells. The membrane was folded so that the pluripotent mesenchymal cellular layer faced the defect and the joint [4] [8]. Between the layers a small amount of sterilized demineralized human bone was placed in a sandwich-like manner. The membranes were fixed to the defects on the femoral condyles using micro bone anchors and fibrin glue. The membrane was fixed to the trochlear defects using fibrin glue alone. The wounds were closed and the sheep were allowed to bear weight as tolerated without immobilization immediately after surgery.

The wounds were assessed for lameness through a clinical evaluation by a licensed veterinarian at designated time intervals of post operatively two weeks, six weeks, and six months. The veterinarian also evaluated for infection and swelling. At six months post-procedure, the sheep were sacrificed and the distal femurs were harvested. Histological samples were taken of each of the operative sites, both those treated with HAM/DMB and without HAM/DMB. Normal cartilage samples were taken from each of the sheep for comparison. The histological samples were taken using mosaicplasty coring instruments to minimize cartilage damage.

The samples were stained with both hemotoxylin and eosin (H \& E) and Trichrome staining. Each of the samples (normal, control, and treated) was evaluated using the O'Driscoll score, which is a straightforward, validated scoring system used in cartilage evaluation [10] [11]. This evaluation method rates the sample on two levels. First, the sample is rated 0 - 3 on overall appearance $(0=$ no cartilage present, $3=$ mostly normal appearing cartilage), which was referred to as "simple score" [12]. Second, each sample was given a percent (\%) value of cartilage-like tissue; these values were referred to as "\% score". All samples were evaluated by both of the senior authors relative to a normal sample of cartilage (Figure 1). The authors were blinded to the results of the other observer.

\section{Statistics}

The results were evaluated by a statistician using SAS 9.3 [13] and Stata 12.1 [14] to determine statistical 


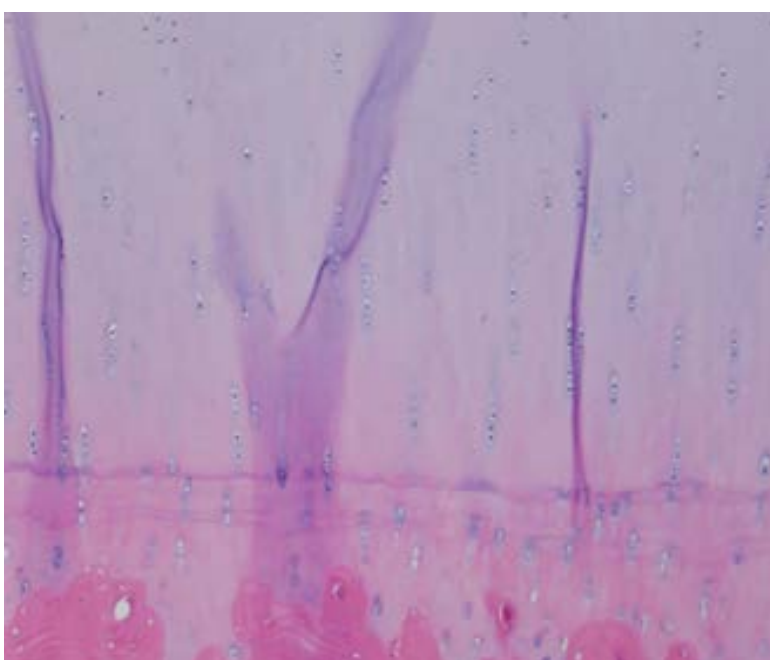

(a)

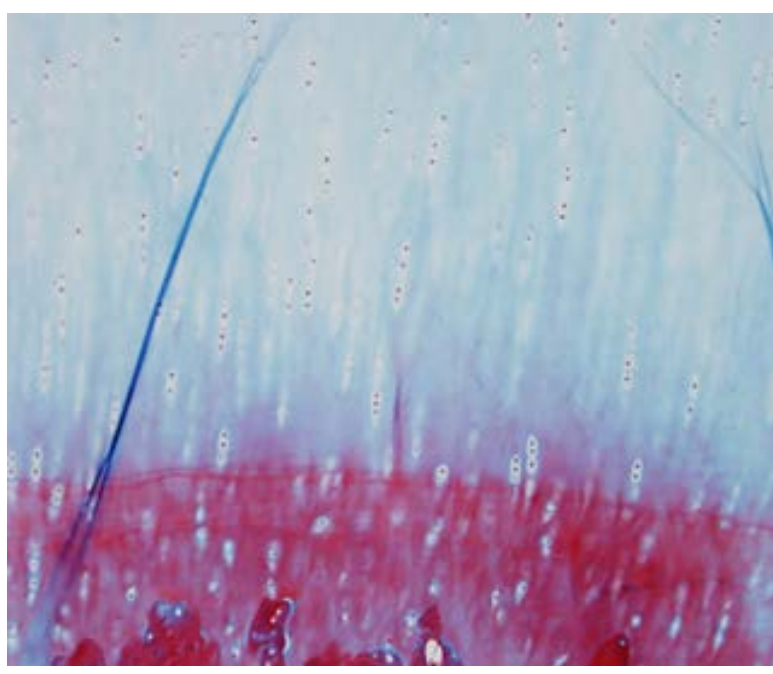

(b)

Figure 1. (a) H \& E stain of "normal” cartilage. (b) Trichrome stain of "normal” cartilage.

significance in: exploratory data analysis, mixed modeling, paired t-tests, signed rank tests, Mantel-Haenszel tests, two-sample $t$-tests, Wilcoxon rank Sum tests, Bland-Altman plots and Spearman rank correlation coefficient analyses.

\section{Results}

When evaluated prior to sacrifice, the sheep indicated recovery from the procedure. Only one sheep (a control) was observed by the large animal veterinarian not to have regained full range of motion (ROM). All sheep, excluding the control without complete ROM, were rated at a lameness level of $0-1$ at final evaluation (Table 1).

Upon gross evaluation, it was found that of the sheep that had HAM/DMB placed in their defects, $50 \%$ retained the membrane grossly. For this reason, analysis of the samples was only performed on those samples that retained the amniotic membrane. All of the retained samples were in the trochlear groove defects and none of the samples on the femoral condyles retained the HAM/DMB.

Upon histological evaluation, the senior authors were found to interpret the HAM/DMB samples with significant correlation. The H \& E and Trichrome stains of the grafts (Figure 2 and Figure 3) and the H \& E stained controls (Figure 4) were compared to the stains of normal cartilage stains (Figure 1) to distinguish the efficacy of the HAM/DMB combination. The simple scores given overall as well as given by each investigator (Table 2) were found to have a relatively strong correlation, $r^{2}=0.79$ (Figure 5(a)). The $\%$ normal appearing cartilage in the grafts, as defined by each investigator as well as overall, was found by each investigator (Table 3). These scores from both of the senior authors have a strong correlation as well, $r^{2}=0.89$ (Figure 5(b)). Additionally, overall comparisons between the physicians' scores were not significantly different, as indicated by Fisher exact test on the simple score $(p=0.26)$ and the Wilcoxon rank sum test on the $\%$ score $(p=0.57$; Table 4$)$. Inter-rater agreement between the physicians was exhibited by the Spearman rank correlation: the values for both simple and \% scores were found to be very high, $\rho=0.94$ and $\rho=0.98$, respectively. The overall mean score difference between physicians was evaluated through a paired $t$-test and a signed-rank test to have p-values of 0.09 and 0.31 , respectively, indicating no statistical difference in scores between paired physician scores (Table 4). Additionally, The Mantel-Haenszel test showed that the distribution of simple scores across the graft types was not significantly different between the two physicians with $\mathrm{p}=0.15$. The kappa statistic of the simple and $\%$ scores between physicians exhibited a moderate to high inter-rater agreement with $\kappa=0.50$ and $\kappa=0.75$ for each, respectively. Furthermore, viewing the Bland-Altman plots, the score difference and mean simple score differences were minimal in both investigators' observations, each score with a standard deviation of 2 (Figure 6).

\section{Discussion}

With only one sheep exhibiting a lack of ROM, despite the lack of immobilization prior to final evaluation, the 
Table 1. All six sheep used in this study at their 2 weeks, 6 weeks, and 6 months evaluations by a large animal veterinarian.

\begin{tabular}{|c|c|c|c|c|}
\hline Sheep & Evaluation & Lameness (0 - 5) & Effusion & ROM \\
\hline \multirow[t]{3}{*}{1 (implant) } & 2 wks & 2 & 3 & \\
\hline & 6 wks & 2 & 0 & \\
\hline & $6 \mathrm{mo}$ & $0-1$ & 0 & full, $\mathrm{nml}$ \\
\hline \multirow[t]{3}{*}{2 (implant) } & 2 wks & 3 & 2 & \\
\hline & 6 wks & 2 & 1 & \\
\hline & $6 \mathrm{mo}$ & $0-1$ & 0 & full, nml \\
\hline \multirow[t]{3}{*}{3 (implant) } & 2 wks & 2 & 2 & \\
\hline & 6 wks & 2 & 0 & \\
\hline & $6 \mathrm{mo}$ & $0-1$ & 0 & full, $\mathrm{nml}$ \\
\hline \multirow[t]{3}{*}{4 (control) } & 2 wks & 3 & 3 & \\
\hline & 6 wks & 1 & 0 & \\
\hline & $6 \mathrm{mo}$ & 2 & 0 & reduced flex \\
\hline \multirow[t]{3}{*}{5 (control) } & $2 \mathrm{wks}$ & 2 & 3 & \\
\hline & 6 wks & 2 & 0 & \\
\hline & $6 \mathrm{mo}$ & $0-1$ & 0 & full, nml \\
\hline \multirow[t]{3}{*}{6 (control) } & 2 wks & 1 & 2 & \\
\hline & 6 wks & 1 & 0 & \\
\hline & $6 \mathrm{mo}$ & $0-1$ & 1 & full, nml \\
\hline
\end{tabular}

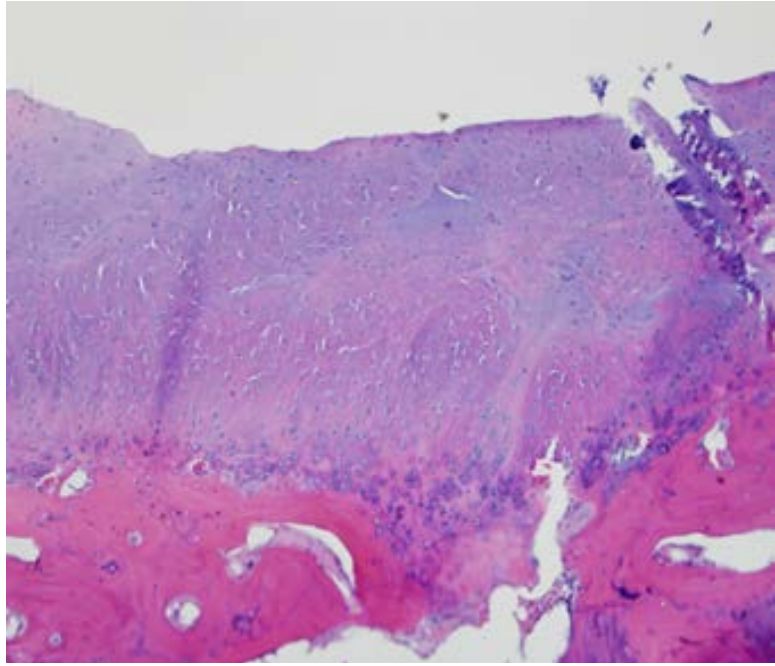

(a)

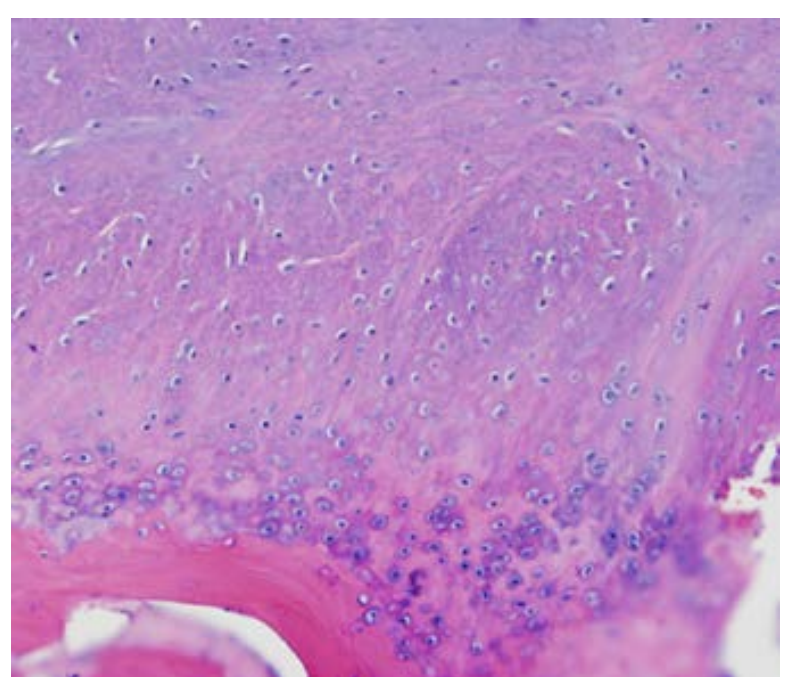

(b)

Figure 2. (a) Low magnification of a HAM/DMB sample of cartilage with $\mathrm{H} \& \mathrm{E}$ staining. (b) High magnification of a HAM/DMB sample of cartilage with $\mathrm{H} \& \mathrm{E}$ staining. 


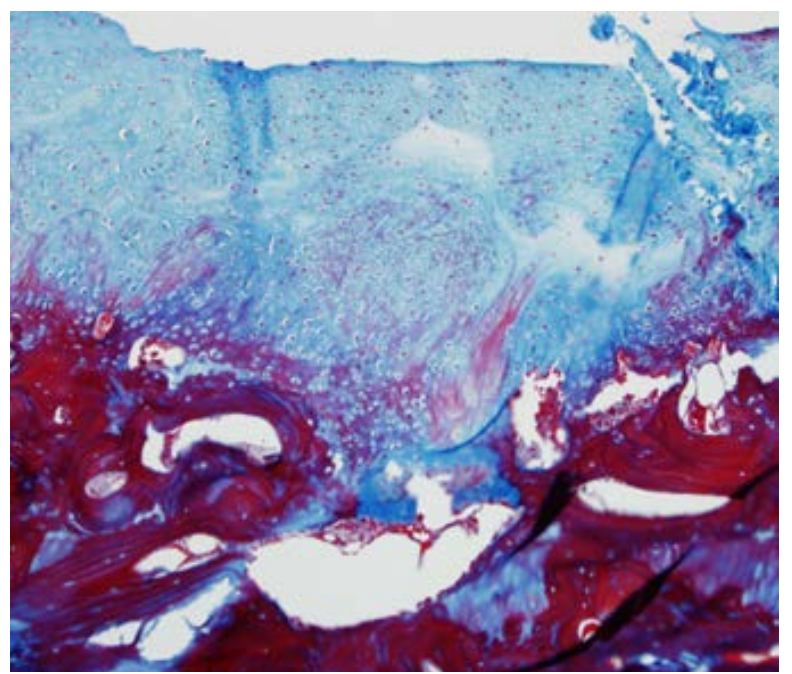

(a)

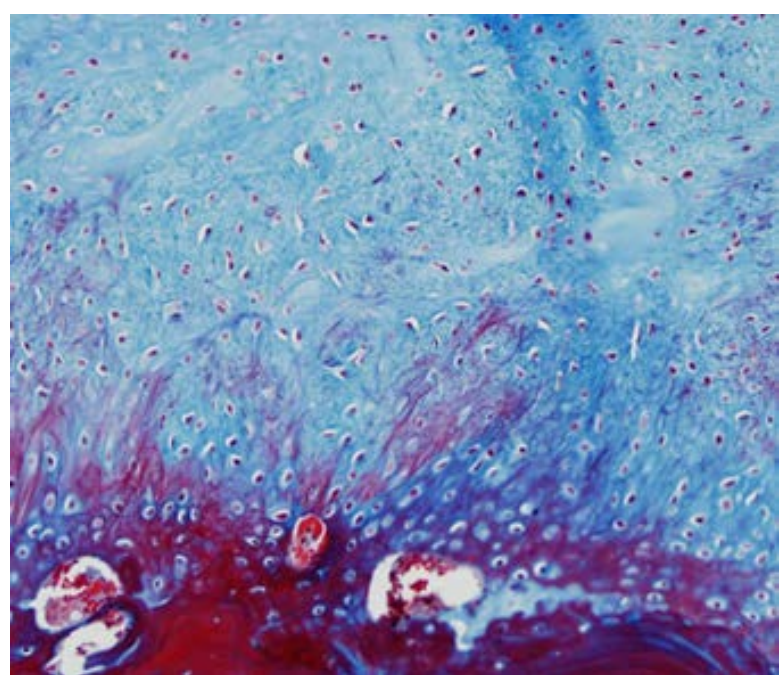

(b)

Figure 3. (a) Low magnification of a HAM/DMB sample of cartilage with Trichrome staining. (b) High magnification of a HAM/DMB sample of cartilage with Trichrome staining.

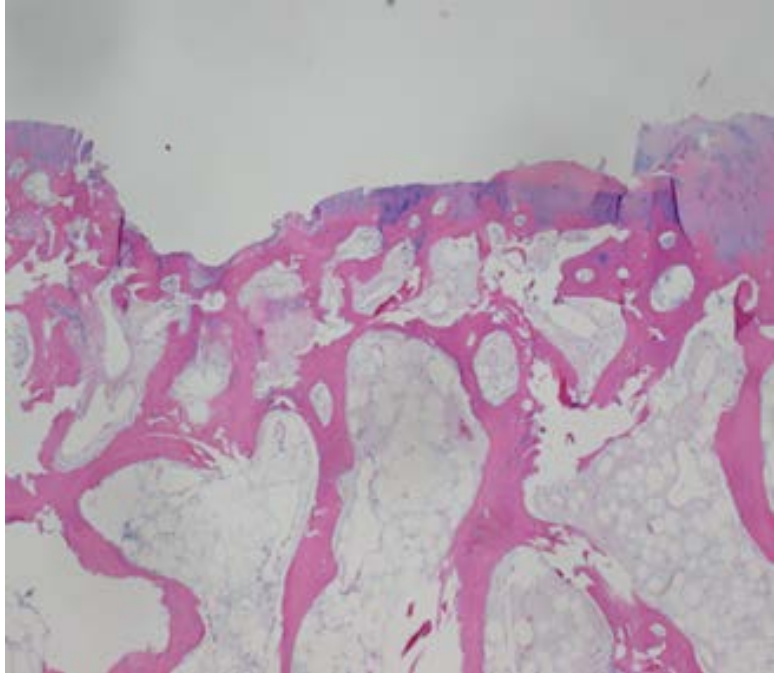

(a)

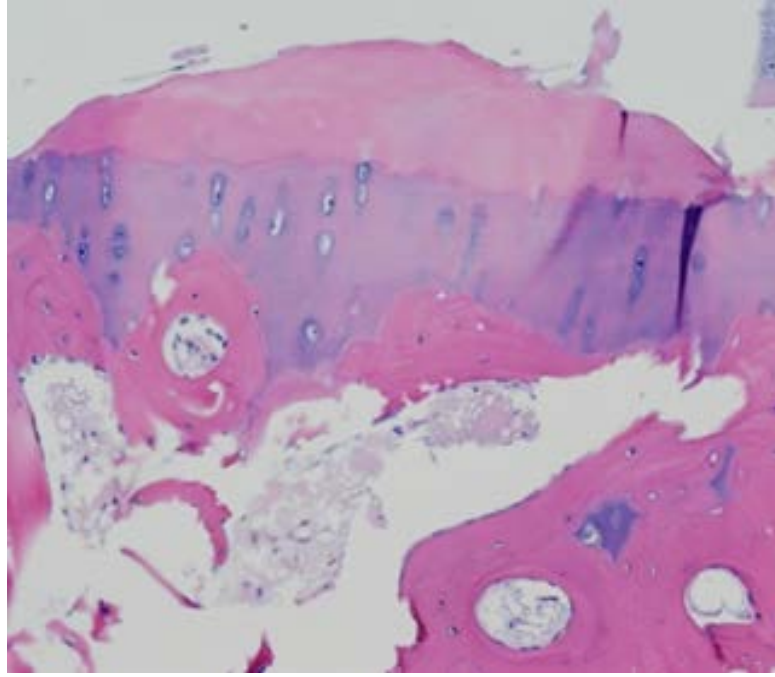

(b)

Figure 4. (a) Low magnification of a control sample of cartilage with H \& E staining. (b) High magnification of a control sample with $\mathrm{H} \& \mathrm{E}$ staining.

filled-in defects remained relatively intact for evaluation after sacrifice. However, because of the loss of $50 \%$ of the grafts (from lack of immobilization), the results were more limited in scope than previously anticipated. With none of the femoral condyles samples remaining at evaluation, the study became confined to results associated only with the trochlear groove samples.

Observing the histological stains of the trochlear samples, the senior authors observed a strong correlation on the simple and \% scores of cartilage in the HAM/DMB grafts (Figure 5). Exhibited by the Mantel-Haenszel, correlation coefficient, Fischer exact test (on simple score), Wilcoxon rank sum (on \% score), Spearman rank correlation, paired $t$-test, signed rank test, and kappa statistic, there is a strong indicator that HAM/DMB has the potential to be influenced to make chondrocytes. As both senior authors determined, with statistical inter-rater agreement, the grafts appeared to be specifically of the mixed hyaline type of cartilage, though further tests of specific staining to highlight the presence of hyaline cartilage may be required in future studies. These correlation statistics clearly indicate a similarity between chondrocytes of the samples and hyaline cartilage. 
Table 2. (a) Overall summary of graft type by simple score by Fisher exact test $(\mathrm{p}<0.0001)$. B) Summary, by physician, of graft type by simple score by Mantel-Haenszel test for difference between physician $(p=0.15)$.

(a)

\begin{tabular}{ccccc}
\hline \multirow{2}{*}{ Graft Type } & \multicolumn{3}{c}{ Simple Score } \\
\cline { 2 - 5 } & 0 & 1 & 2 & 3 \\
\hline Control & $2(8 \%)$ & $16(67 \%)$ & $6(25 \%)$ & $0(0 \%)$ \\
Graft & $0(0 \%)$ & $0(0 \%)$ & $0(0 \%)$ & $24(100 \%)$ \\
Normal & $0(0 \%)$ & $0(0 \%)$ & $0(0 \%)$ & $48(100 \%)$ \\
\hline
\end{tabular}

(b)

\begin{tabular}{ccccccccc}
\hline & \multicolumn{9}{c}{ Simple Score } \\
\cline { 2 - 8 } Graft Type & \multicolumn{9}{c}{ Conner } & \multicolumn{3}{c}{ Tabet } \\
\cline { 2 - 9 } & 0 & 1 & 2 & 3 & 0 & 1 & 2 \\
Control & $1(8 \%)$ & $6(50 \%)$ & $5(42 \%)$ & $0(0 \%)$ & $1(8 \%)$ & $10(83 \%)$ & $1(8 \%)$ & $0(0 \%)$ \\
Graft & $0(0 \%)$ & $0(0 \%)$ & $0(0 \%)$ & $12(100 \%)$ & $0(0 \%)$ & $0(0 \%)$ & $0(0 \%)$ & $12(100 \%)$ \\
Normal & $0(0 \%)$ & $0(0 \%)$ & $0(0 \%)$ & $24(100 \%)$ & $0(0 \%)$ & $0(0 \%)$ & $0(0 \%)$ & $24(100 \%)$ \\
\hline
\end{tabular}

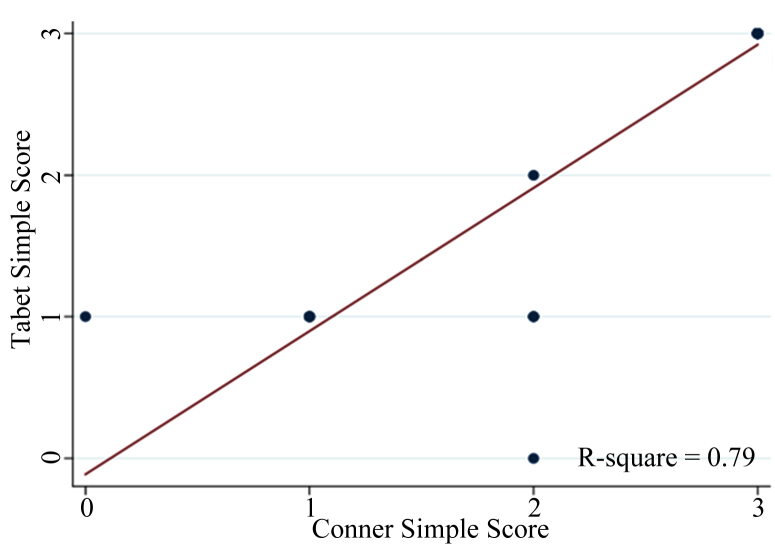

(a)

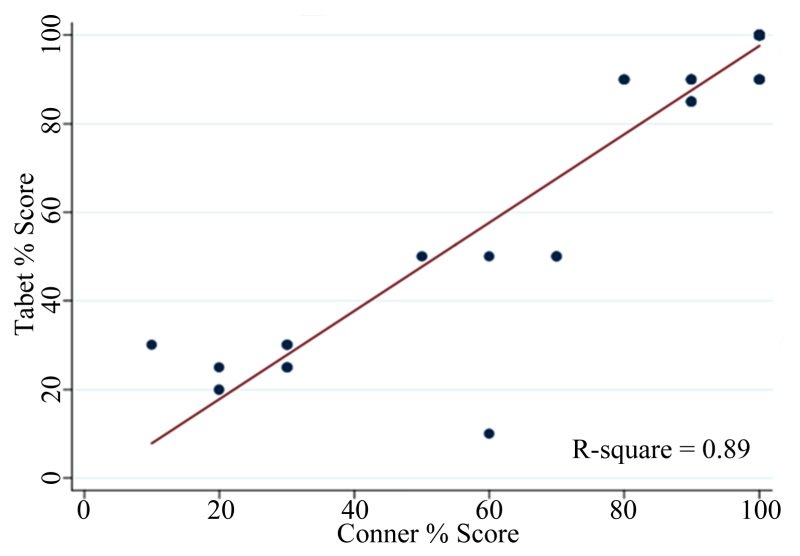

(b)

Figure 5. (a) Scatter plot of simple scores to demonstrate the relationships between physicians where $r^{2}=0.79$. (b) Scatter plot of \% scores to demonstrate the relationship between physicians where $r^{2}=0.89$.

Table 3. (a) Overall summary of graft type by \% score. (b) Summary, by physician, of graft type by \% score.

(a)

\begin{tabular}{cccc}
\hline & & \multicolumn{3}{c}{ \% Score } \\
\cline { 2 - 4 } Graft Type & $\mathrm{n}$ & Mean & $95 \% \mathrm{Cl}$ \\
\hline Control & 24 & 36.5 & $(29.0,43.9)$ \\
Graft & 24 & 89.2 & $(87.2,91.1)$ \\
Normal & 48 & 99.8 & $(99.4,100.2)$ \\
\hline
\end{tabular}

(b)

\begin{tabular}{ccccccc}
\hline & \multicolumn{9}{c}{ Simple Score } \\
\cline { 2 - 7 } Graft Type & \multicolumn{9}{c}{ Conner } & \multicolumn{2}{c}{ Tabet } \\
\cline { 2 - 7 } & $\mathrm{n}$ & Mean & $95 \% \mathrm{Cl}$ & $\mathrm{n}$ & Mean & $95 \% \mathrm{Cl}$ \\
\hline Control & 12 & 40.0 & $(26.7,53.3)$ & 12 & 32.9 & $(24.2,41.6)$ \\
Graft & 12 & 90.0 & $(86.2,93.8)$ & 12 & 88.3 & $(86.8,89.9)$ \\
Normal & 24 & 100.0 & -- & 24 & 99.6 & $(98.7,100.4)$ \\
\hline
\end{tabular}


Table 4. (a) Overall simple score by physician by Fisher exact test ( $\mathrm{p}=0.26)$. (b) Overall \% score by physician by Wilcoxon rank sum test $(\mathrm{p}=0.57)$. (c) Overall mean score difference between physicians by 1 ) paired t-test and 2 ) signed-rank test.

(a)

\begin{tabular}{ccccc}
\hline & \multicolumn{3}{c}{ Simple Score } \\
\cline { 2 - 5 } Physician & 0 & 1 & 2 & $36(75 \%)$ \\
Conner & $1(2 \%)$ & $6(13 \%)$ & $5(10 \%)$ & $36(75 \%)$ \\
Tabet & $1(2 \%)$ & $10(63 \%)$ & $1(2 \%)$ & \multicolumn{3}{c}{. } \\
\hline
\end{tabular}

(b)

\begin{tabular}{cccc}
\hline & & \multicolumn{2}{c}{ \% Score } \\
\cline { 2 - 4 } Physician & $\mathrm{n}$ & Median & Q1,Q3 \\
\hline Conner & 48 & 100.0 & $(75.0,100.0)$ \\
Tabet & 48 & 90.0 & $(67.5,100.0)$ \\
\hline
\end{tabular}

(c)

\begin{tabular}{ccccccc}
\hline & \multicolumn{5}{c}{ Difference } & Q-value \\
\cline { 2 - 5 } Physician & $\mathrm{n}$ & Mean & $95 \% \mathrm{Cl}$ & Median & Q1,Q2 & 0.091 \\
\% Score & 48 & 2.4 & $(-0.3,5.1)$ & -- & -- & 0.312 \\
Simple Score & 48 & -- & -- & 0.0 & $(0.0,0.0)$ & 0.00 \\
\hline
\end{tabular}

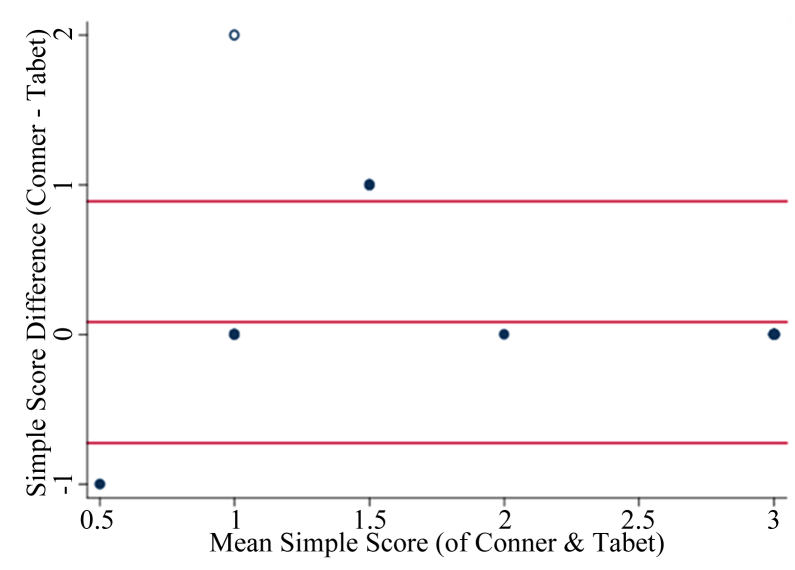

(a)

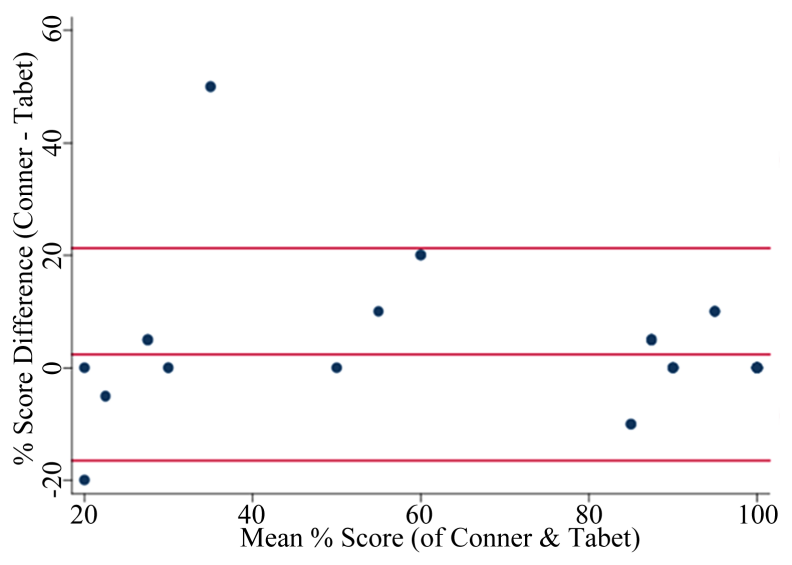

(b)

Figure 6. (a) A Bland-Altman plot for simple score difference between physicians where reference lines are the mean difference $+/-2$ standard deviations. (b) A Bland-Altman plot for mean \% score of physicians where reference lines are mean difference $+/-2$ standard deviations.

This study promotes promising results: human amniotic membrane is a potential source of pluripotent cells that can be influenced to produce specifically hyaline-like cartilage in sheep models. The implications for application in a human model are promising, potentially providing an alternative to microfracture or abrasion techniques of varying efficacy. The expectations are realistic, as many studies have already shown the potential uses of human amniotic cells in various platforms of tissue repair [1]-[4]. Harnessing the pluripotency of human amniotic cells to produce a stroma rich in chondrocytes and much like normal hyaline cartilage is potentially the next step in providing surgeons with a greater number of options for the varying degrees of surgical intervention needed.

Future modifications to this study would be to increase the sample size to incorporate greater statistical power with which to make deductions, especially if all femoral grafts dislodge again. Missing from this study was the 
utilization of immunostaining techniques to quantitate levels of collagen I and collagen II in the samples. In addition, in an effort to monitor the sheep models more effectively, magnetic resonance imaging is advised periodically before sacrificing the sheep. Incorporating these changes will lend greater clarity on the already promising concept of using DMB with HAM to incite hyaline cartilage restoration in sheep models and eventually human patients. Due to the current lack of literature on the subject, it is difficult to evaluate the consistency of the overall results against similar studies. However, the histology results are consistent with the current amniotic membrane literature showing promising results in tissue repair [1]-[4].

\section{Acknowledgements}

We would like to thank Dr. Douglas Thal for assisting with procuring the sheep for this study and ensuring the ethical treatment of these animals. Additionally, we appreciate NuCel Inc. for funding this study and New Mexico Orthopaedics Associates for allowing lab space for experimental procedures associated with this study. With regard to analysis of results, we are grateful for Cristina Murray-Krezan’s statistical contributions.

\section{References}

[1] Boo, L., Sofiah, S., Selvaratnam, L., Tai, C., Pingguan-Murphy, B. and Kamarul, T. (2009) A Preliminary Study of Human Amniotic Membrane as a Potential Chondrocyte Carrier. Malaysian Orthopaedic Journal, 3, 16-23. http://dx.doi.org/10.5704/MOJ.0911.004

[2] Akle, C.A., Adinolfi, M., Welsh, K.I., Leibowitz, S. and McColl, I. (1981) Immunogenicity of Human Amniotic Epithelial Cells after Transplantation into Volunteers. The Lancet, 318, 1003-1005. http://dx.doi.org/10.1016/S0140-6736(81)91212-5

[3] He, H., Li, W., Tseng, D., Zhang, S., Chen, S., Day, A. and Tseng, S. (2009) Biochemical Characterization and Function of Complexes Formed by Hyaluronan and the Heavy Chains of Inter- $\alpha$-inhibitor (HC-HA) Purified from Extracts of Human Amniotic Membrane. Journal of Biological Chemistry, 284, 20136-20146. http://dx.doi.org/10.1074/jbc.M109.021881

[4] Marongiu, F., Gramignoli, R., Sun, Q., Tahan, V., Miki, T., Dorko, K., Ellis, E. and Strom, S.C. (2010) Isolation of Amniotic Mesenchymal Stem Cells. Current Protocols in Stem Cell Biology, 12, 1E5.1-1E5.11. http://dx.doi.org/10.1002/9780470151808.sc01e05s12

[5] Jin, C.Z., Park, S.R., Choi, B.H., Lee, K., Kang, C.K. and Min, B.H. (2007) Human Amniotic Membrane as a Delivery Matric for Articular Repair. Tissue Engineering, 14, 693-702. http://dx.doi.org/10.1089/ten.2006.0184

[6] Zhou, S., Yates, K.E., Eid, K. and Glowacki, J. (2005) Demineralized Bone Promotes Chondrocyte or Osteoblast Differentiation of Human Marrow Stromal Cells Cultured in Collagen Sponges. Cell and Tissue Banking, 6, 33-44. http://dx.doi.org/10.1007/s10561-005-4253-y

[7] Kanthan, S.R., Ganesan, K., Azura, M. and Kamarul, T. (2010) The Different Preparations of Human Amniotic Membrane (HAM) as a Potential Cell Carrier for Condrocytes. European Cells and Materials, 20, 46.

[8] Steigman, S.A. and Fauza, D.O. (2007) Isolation of Mesenchymal Stem Cells from Amniotic Fluid and Placenta. Current Protocols in Stem Cell Biology, 1E.2.1-1E.2.12. http://dx.doi.org/10.1002/9780470151808.sc01e02s1

[9] Lindenmair, A., Wolbank, S., Stadler, G., Meinl, A., Peterbauer-Scherb, A., Eibl, J., Polin, H., Gabriel, C., Van Griensven, M. and Redl, H. (2010) Osteogenic Differentiation of Intact Human Amniotic Membrane. Biomaterials, 31, 8659-8665. http://dx.doi.org/10.1016/j.biomaterials.2010.07.090

[10] O’Driscoll, S.W., Marx, R.G., Beaton, D.E., Miura, Y., Gallay, S.H. and Fitzsimmons, S.H. (2002) Validation of a Simple Histological-Histochemical Cartilage Scoring System. Tissue Engineering, 7, 313-320. http://dx.doi.org/10.1089/10763270152044170

[11] Moojen, D.J.F., Saris, D.B.F., Yang, K.G., Dhert, W.J.A. and Verbout, A.J. (2002) The Correlation and Reproducibility of Histological Scoring Systems in Cartilage Repair. Tissue Engineering, 8, 627-634. http://dx.doi.org/10.1089/107632702760240544

[12] Grogan, S.P., Barbero, A., Winkelmann, V., Fitzsimmons, J., O’Driscoll, S., Martin, I. and Mainil-Varlet, P. (2006) Visual Histological Grading System for the Evaluation of in Vitro-Generated Neocartilage. Tissue Engineering, 12, 2141-2149. http://dx.doi.org/10.1089/ten.2006.12.2141

[13] (2012) SAS 9.3. SAS Institute Inc., Cary. www.sas.com

[14] (2012) STATA/SE 12.1. Statacorp LP, College Station. www.stata.com 